\title{
Power Consumption for Routing Improvement using AODV_EXT \& AODV_EXT_BP
}

\author{
M. Prasad, M. Sreenivasu, N. Lakshmi, A. Mallikarajun Reddy
}

\begin{abstract}
Basically, in Mobile Ad-hoc NETworks (MANETs), portable hubs are used to control the remote connections in a multiple way. So this system is based on the connection of hub, which transfers data from one packet to another packet. In proposed system, nodes connected to the hub will host the capacity of system.Because of this there will be no loss of communication. This will expand the life time of the network using imperative factors. The ad-hoc network gives minimum number of bounces after the routing process. Coming to energy aware routing protocol, it reduces the residual energy in effective way. In this paper $A O D V_{-} E X T$ and $A O D V_{-} E X T \_B P$ protocols are proposed. The proposed protocols will be analyzed and produces results in effective manner compared to others. So, this system gives low energy consumption.
\end{abstract}

Key Words-Mobile ad-hoc network, Routing message overhead, Route discovery, Broadcast, Bayesian probability, Flooding, Power-aware routing, Routing protocols, AODV, $A O D V_{-} E X T, A O D V_{-} E X T_{-} B P$.

\section{INTRODUCTION}

A specially appointed system is a promising innovation which can be connected in a broad number of regions extending from natural observing to fiasco the executives. Moreover, specially appointed systems can be executed in use of sensors for procedure mechanization in a decent variety of modern applications. Occasions, for example, Routing network will often serve to represent the defect of midway overseen systems and therefore the significance of innovative add imprompt systems, for instance, versatile specially appointed systems (MANETs), wherever a brought along network is not needed. Magnet could be a remote system that includes of versatile hubs with no pre-decided foundation. One among the numerous limitations of impromptu system frameworks are that the vitality accessibility and the extent of gadgets which suggests that management with vast lifetime.

Aside from the activity of domestically out there hardware, management utilization depends upon varied procedures and overheads needed to appear once accessibility. There square measure varied prescribed answers for arrangement with accessibility problems and power restriction in ad-hoc systems. Such ways contain advancement of apparatus, steering calculations, conventions and battery innovation or power the board frameworks. There square measure associatealysts that have

Revised Manuscript Received on August 19, 2019.

M. Prasad, Professor, CSE Department, BVCITS, Amalapuram (E-mail: prasads.maddula@gmail.com)

M. Sreenivasu, Associate Professor, CSE Department, GIET CE, Rajamahendravaram.(E-mail: morukurthi.sreenivasu@gmail.com)

N. Lakshmi, Associate Professor, MCA Department, BVC ITS, Amalapuram.(E-mail: n.lakshmi27@gmail.com)

A. Mallikarajun Reddy, Professor, CSE Department, Anurag Group of Institutions.(E-mail: mallikarjunreddycse@cvsr.ac.in) suggested the advancement of an improved instrumentality which may be used in applications enthusiastic about data rates. There's a proposition to regulate vitality management to applications wherever the voltage, and during this manner handling speed and power, will be diminished for non-time touchy applications.

Totally different techniques that are projected square measure gone for keeping far-away from system apportionment by dominant force utilizations of basic affiliation hubs. As of late, various probabilistic methodologies are projected with the shall lighten the flooding surprise and pay attention of the communicate storm issue.

New routing algorithms square measure needed keeping in mind the top destination to trot out the overhead of skillfulness and topology changes in such energy compelled surroundings. The accidental On Demand Separation Vector (AODV) routing protocol is planned to be used by mobile nodes in a poster hoc network. It offers brisk adaptation to dynamic affiliation conditions, low getting ready and memory overhead, low network usage, and decides unicast courses to destinations within the ad hoc network .In this paper, associate economical energy conscious routing protocol is projected, that is visibleof the on-request accidental routing protocol AODV, and decides a legitimate manner considering node residual battery powers.

The projected protocol plans to expand the time period of the overall detector network by utilizing each, bounce take into account well as node residual battery power. Wireless communication technologies incorporate world System for Mobile Communication (GSM), satellite communication, Wireless native space web layer (WLANs) and Worldwide ability for Microwave Access.

\section{BACKGROUND}

In present generation wireless systems are most widely used for effective response of systems. Their applications keep running from essential remote uninformed rate transmitting sensors [10] to high data rate consistent structures. Most of the present versatile remote development relies upon point-to-point advancement. A point of reference is the Global System for Mobile communication with a building that relies upon adaptable centers examining direct with central paths. Sometimes there are frameworks that don't rely upon united accessibility, for instance, Mobile Ad Hoc Networks (MANET).

Published By: 
MANET is a remote framework that has convenient center points with no fixed establishment. These sorts of frameworks are used in domains, for instance, regular checking or in rescue exercises. The essential confinement of off the cuff systems is the openness of power. The fundamental goals of this examination are to assess existing conventions and directing calculations for power productivity and, distinguish and create procedures (convention as well as steering calculation) that will improve specially appointed system control effectiveness.

Remote correspondence has turned out to be one of the quickest developing advances worldwide because of the innovative progressions in portable processing gadgets and remote innovation. Cell phones, for example, PCs, net books and cell phones have decreased in size but then have huge recollections and high preparing force. Remote correspondence systems give an assortment of points of interest over conventional wired systems. Right off the remote systems permit anyplace and whenever availability and can be sent in zones with existing communication systems or wherever it is hard to setup a wired system. Models incorporate where the law precludes the establishment of links, for example, in legacy structures. What's more the establishment of a remote system is a lot less expensive than a wired foundation making remote systems an alluring arrangement, particularly in less created world. Moreover, remote systems give adaptability and empower simple correspondence setup. For example, versatile clients can turn on their PCs and quickly interface with the Internet in open places, for example, college grounds, bistros or airplane terminals.

A blend of joined and off the cuff frameworks is envisioned to give answers for the game plan of ubiquitous correspondence for a wide extent of employments. While brought together correspondence is settled in, uncommonly designated frameworks organization is seen as the course forward for self dealing with and supervising frameworks which murder cautious and exorbitant masterminding, amazing cost, unyielding nature and frailty inborn in fixed halfway regulated frameworks, for instance, wired and remote frameworks for instance Versatile Telecommunications System. Uniquely selected frameworks also hold unimaginable assurance and applications in a wide number of domains running from calamity the administrators to natural checking.

Headway in extemporaneous frameworks is also promising the use of sensors for methodology computerization in a grouping of endeavors and is engaging advancement in sensor mix. Flighty events, for instance seismic tremor consistently serve to depict the lack of protection of midway regulated frameworks and the essentials of inventive work in exceptionally delegated frameworks, for instance, Mobile Ad-hoc Networks (MANET), for which bound together accessibility isn't required. MANET is a remote framework that has convenient center points with no fixed establishment.

The essential requirement of off the cuff framework structures is the availability of power and relentless lessening in the proportion of devices suggest that control obstruction can't simply be improved with immense battery packs. Despite running the introduced contraptions, control usage is spoken to by the amount of systems and overheads required to take care of accessibility. A wide extent of frameworks has been proposed to address accessibility and power limitation issues in exceptionally selected frameworks. These join gear progression, shows, controlling counts and battery development or imperatives the board systems.

A couple of masters have proposed the improvement of gear upgraded for unequivocal applications subject to data rates. Dynamically point by point examinations of essentials use by gear have been finished to evaluate imperatives use when transmitting, getting, in rest and idle modes. It has been recommended that imperatives load up should be specially fitted to each application where the voltage, and in this manner taking care of pace and essentials. The common target of imperatives the administrators frameworks proposed for uniquely selected frameworks is to shield essentials and lift life length of the framework. Other proposed methods are away for checking framework dividing directing essentials uses of fundamental association centers. In this paper we propose a difference in a champion among the most comprehensively used shows to improve the essentials and data transmission adequacy of the framework.

\section{REALTED WORK}

The principle routing conventions square measure of 2 types as example protocol activation sort and Network structure sort. Leading convention are often organized in to 3 categories above all, proactive, responsive and half and half convention relying upon however the supply sends a course to the goal. In proactive (table-driven) steering convention; knowledge communicate to every neighboring hub. each hub keeps knowledge regarding neighborhood hubs, accessible hub and therefore the amount of jumps in their separate leading table. Each hub unendingly continue course between try of hubs. Responsive (on-request) this leading convention known as On-request convention since courses square measure engineered au fait interest as they're needed. Once approach settled; a course is continue as long because it is needed.

Routing protocol [1], [2] got to be appropriate wear down a considerable variety of hubs with forced assets. The first issue partner with the steering convention includes being showed up and nonexistent of hubs in several areas. It's need to diminish steering message overhead in spite of the event variety of hubs. Steering convention desires following characteristics to be compelling: disseminated task, circle chance, request primarily based activity, proactive activity, security and unidirectional affiliation support. Circulated task implies that any hub will enter or leave at no matter purpose they have. Loop is to anticipate overhead created amid sending knowledge pointlessly. Request primarily based activity is to diminish traffic and use transmission capability assets all the additional proficiently. Proactive task is employed after they need enough knowledge transfer capability and vitality assets. 
Security is that the most imperative issue for any correspondence.Steering convention is assessed supported however and once course square measure found, nevertheless each choose the foremost temporary thanks to the goal. Coming to the proactive routing protocol, it utilizes connect state leading calculations that floods interface knowledge regarding its neighbors as typically as potential. This type of convention stays up with the newest leading knowledge between every try of hubs by causation management message sometimes in system. one in every of the elemental favorable circumstances of this convention is that courses square measure ready to utilize once needed. The disadvantage of proactive steering conventions incorporates the overhead of flooding course.

Responsive or on-request steering conventions were supposed to reduce overheads gift in proactive conventions by taking care of knowledge. It utilizes separate vector leading calculation and sets up the course to given goal simply once a hub demand it by beginning course revelation method. This conventions chip away at course revealing and course maintenance element. Receptive leading conventions have disadvantage of deferral in discovering courses to new assets or goal.

Hybrid routing protocol could be a system steering convention that joins separate vector leading convention. What is additional, connect state steering convention highlights. HRP is employed to make a decision ideal system goal course and report prepare topology info amendment. Cross breed leading sometimes alluded to as adjusted crossover steering. of versatile hubs. For assessment This paper centers around 3 of the parameters specifically traffic rate, speed and respite time reason the execution measurements utilized square measure output, Bandwidth, traditional finish - to finish delay, Packet Delivery Ratio (PDR) and Normalized Overhead Load (NOL). Assess the AODV convention for QoS conjointly Non QoS is finished with thence the parameters.Execution measurements [3] by strategies for graphical portrayal of their interrelations.

AODV offers low system use and uses goal arrangement variety to ensure circle chance. It's a responsive convention inferring that it demands a course once needed and it does not continue courses for those hubs that do not effectively take an interest in an exceedingly correspondence. A for the foremost half highlight of AODV is that it utilizes a goal succession variety, that relates to a goal hub that was mentioned by a steering sender hub. The goal itself furnishes the amount aboard the course it must go for reach from the solicitation sender hub up to the goal. On the off probability that there square measure totally different courses from a solicitation sender to a goal, sender takes the course with the next grouping variety. This guarantees the specially appointed system convention remains circle free. Impromptu On-request Distance Vector (AODV) steering convention is that the standout amongst the foremost prevailing leading convention for versatile specially appointed systems. This work upgrades AODV [4] convention by limiting the combination of burden and deferral.

AODV could be a separation vector routing protocol which fits beneath the classification of proactive leading

convention that depends on ancient Bellman-Ford steering calculation. This convention includes another property, grouping variety, to each course table passage at every hub. Steering table is preserved at one hub and with this table, hub transmits the bundles to other nodes within the system. This convention was persuaded for the employment of knowledge trade on dynamical [8] and self-assertive ways in which of interconnection which cannot be close to any base station.

There is no static foundation for the system, as an example, a server or a base station. The chance of such systems administration is to assist powerful and effective task in moveable remote systems by fusing steering utility into versatile hubs. A specially appointed system, wherever there square measure varied mixes of transmission zones for varied hubs [5].

From the supply hub to the goal hub, there are often various ways in which of association at a given purpose of your time. In any case, each hub additional typically than not contains a forced zone of transmission. In an advertisement hoc versatile system [5], every hub within the system conveys its terribly own switch with it, and every one hub coordinate in convincing traffic. The whole logic of the unintentional systems administration show is an extreme takeoff from the terribly organized and each currently and once more varied leveled models utilized for each neighborhood wide region organizing, at the moment being employed.

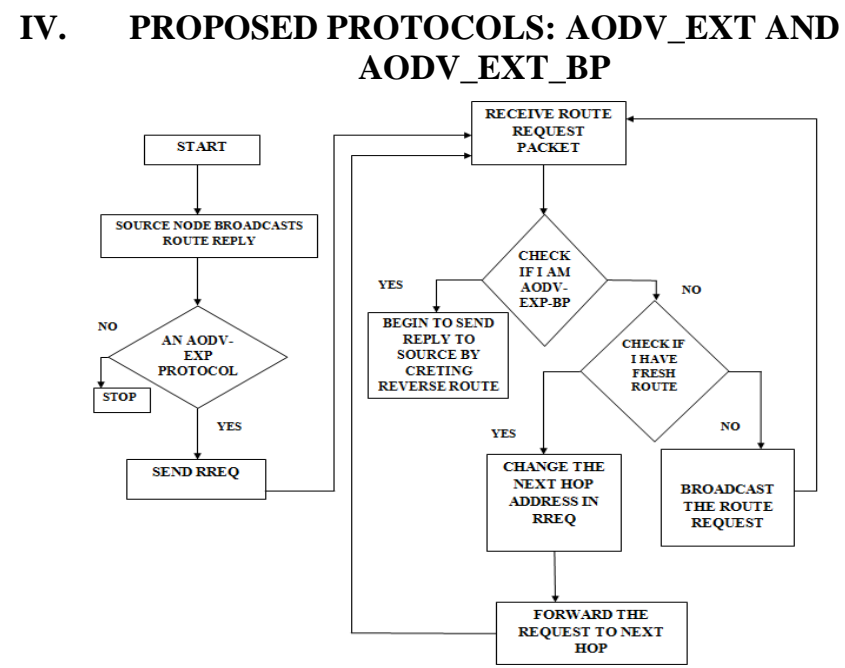

Fig. 1: PROPOSED FLOW CHART

The above Fig. 1 shows the flow chart of proposed system. In this first we will start the process by sending source node broadcast route reply to AODV_EXT protocol to check if it is AODV_EXT protocol or not. If it is AODV_EXTprotocol then it sends a route request packet to the receiver. If it is not AODV_EXT protocol then the entire function will stops. Now the receiver receives the protocol and check if it is AODV_EXT_BP. Now, if it is AODV_EXT_BP then it starts the process of creating reverse route. If it is not a AODV_EXT_BP protocol then it will check again for fresh route. 
If fresh route is available then it changes the hop address in route request packet. If fresh route is not available then it broadcast the route request. After changing the hop address of route request packet, it will forward the request to next hop. So in the same way the operation is performed. So, this proposed system will process the operation very quickly and reduce the delay [6] compared to other system. The below shows the detailed explanation of proposed protocols.

\section{A. AODV_EXT PROTOCOL}

Basically, the proposed AODV_EXT protocol will send request to all centre points in the system. After request is sent one centre point will interact with other centre point. So, after interacting with each other, the message will transmits to the centre in zone. Now depend on the nature of organization, the zones will be contrasted dynamically.

But here the message will be accepted by the centre point in a zone through a condition i.e., ; the interaction of centre points will relay upon the thickness of neighborhood centre point. So at last the limit of proposed system excess depends on transmission.

In AODV_EXT characterizes a base condition which gives more noteworthy conviction of effectively parcels directing however limits excess transmissions. Additionally this implies on the off chance that there are five hubs or less in the entire system, at that point the calculation picks the flooding strategy that the first form of AODV utilizes so as to communicate the RREQ parcels.

An arbitrary number generator is utilized to produce a succession of numbers somewhere in the range of 0 and 100 . Utilizing an irregular number generator lessens the opportunity of utilizing a similar middle of the road hubs between the source and the goal hub. In this proposed calculation it produces irregular numbers somewhere in the range of 0 and 100 . On the off chance that the RREQ is gotten from a middle hub, at that point there will be no less than one conceivable way which incorporates that hub in its way list. More prominent vitality investment funds could be accomplish by utilizing a separation subordinate system, a procedure that tallies the base number of hubs on the most limited directing way so as to gauge the separation between two hubs. In AODV_EXT conventions, the coordinating administrator controls every datum pack for source-course information.

The bundles are then sent by the controlling information. After the information is sent, then the source course will offer a pack to control the system. When the objective isn't understood it stores the pack and conveys course questions. The controlling request is at first sent to all the neighboring center points and then enabled by using data pack. Course answers are sent back if the data with respect to the course to the goal is required. AODV_EXT devours less power than OLSR (Optimized Link State Routing Protocol) and DSDV (Destination-Sequenced Distance-Vector Routing) conventions. For AODV convention it has nearly a similar conduct with AODV_EXT until the thickness of the system increments to in excess of 40 hubs where it expends more vitality than the AODV_EXT. The DSR convention devours more vitality contrasting with AODV and AODV_EXT however indicates better execution when the thickness of the system is medium (30 hubs).

\section{B.AODV_EXT_BP PROTOCOL}

In this protocol Bayesian probability is used to alleviate the flooding issue in AODV. The routing updates are enabled in this protocol. Here the routing data will transform from one form to another form to update the procedure.Because of this update procedure there will be adjustment in directing table through contagious hubs. Now the directing inquiries will be reserved in the form of packet. These packets are stored in a hub. Here the packets are dropped depend upon the particular size and time restriction.Here packets are steered specifically when they stored in conveyed manner. Sometimes hub doesn't creates the location to save the packets, in such case the operator will direct the packets and send it to the default hub.

In this system diverse components and calculations are utilized to direct the convection. At last routing procedure will produce a communication format in straight forward flooding way. The request protocol is transferred from source hub to system. Re-transmission process is obtained with high clog and impacts in the system. Compared to other system, the request forwarding system will reduces the overhead in the system. The probability of request protocol reduces the energy consumption [7], [9]. To evaluate the routing protocols, we use AODV_EXT_BP protocol is used.

\section{RESULTS}

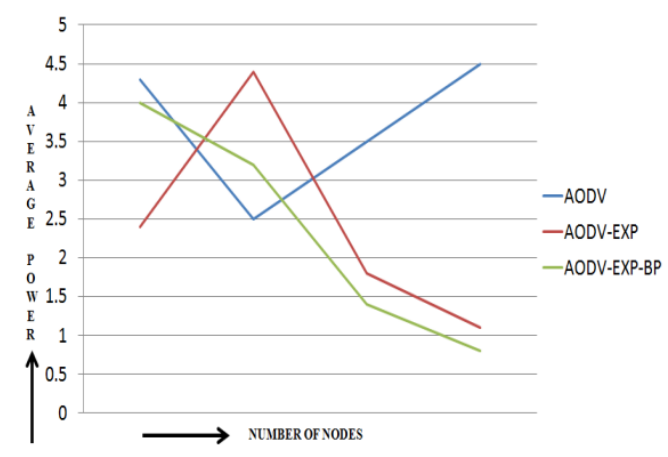

Fig, 2: THE AVERAGE REMAINING BATTERY POWER

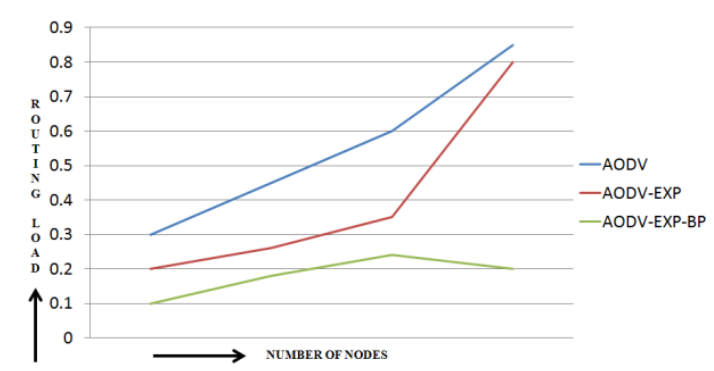

Fig, 3: ROUTING LOAD AGAINST THE NUMBER OF NODES

\section{CONCLUSION}

A mix of unified and specially appointed systems is imagined to give answers for the arrangement of communication for a wide scope of utilizations. Here a route request protocol is used to send message in AODV system.

Published By:

Blue Eyes Intelligence Engineering 
Two likelihood techniques have been realized in the RREQ sending instrument of AODV to diminish retransmissions. These are: AODV_EXT, and the AODV_EXT_BP. The proposed calculations help the system to reduce the total system control utilization.

\section{REFERENCES}

1. Myung Jong Lee, JianlingZheng, Xuhui Hu, Hsin-hui Juan, Chunhui Zhu, Yong Liu, June Seung Yoon, and Tarek N. Saadawi, "A New Taxonomy of Routing Algorithms for Wireless Mobile Ad Hoc Networks: The Component Approach", IEEE Communications Magazine, vol. 44, no.11, pp. 116-123, November 2006.

2. K. Akkaya and M. Younis, "A Survey of Routing Protocols in Wireless Sensor Networks", Elsevier Ad Hoc Network Journal, Vol 3/3,pp.325-349, 2005

3. VeenaVenugopa, RadimBarto, Michael J. Carter, and Sai S. Mupparapu, "Improvement of Robustness for Ad Hoc Networks Through Energy-Aware Routing", University of New Hampshire, Durham, NH 03824, USA,2002.

4. Charles E. Perkins, "Ad hoc On-Demand Distance Vector (AODV) Routing”, INTERNET DRAFT, Mobile Ad Hoc Networking Working Group, 17 February 2003.

5. Bor-rong Chen and C. Hwa Chang, "Mobility Impact on Energy Conservation of Ad Hoc Routing Protocols", TuftsUniversity Medford, MA 02155, U.S.A,2001.

6. SampoNaski, "Performance of Ad Hoc Routing Protocols: Characteristics and Comparison", Seminar on Internetworking Sjokulla, 2004

7. R. Min, A. Chandrakasan, "Top Five Myths about the Energy Consumption of Wireless Communication", Mobile Computing and Communications Review, Vol. 7, No. 1, 2003

8. R. Ramdhany, P. Grace, G. Coulson, D. Hutchison, "Dynamic Deployment and Reconfiguration of Ad hoc Routing Protocols", Journal of Internet Services and Applications, Vol. 1, No. 2, pp. 135$152,2010$.

9. Y. Xu, J. Heidemann, D. Estrin, “ Geography-informed Energy Conservation for Ad Hoc Routing", Proceedings of the Seventh Annual ACM/IEEE International Conference on Mobile Computig and Networking, Italy, 2001.

10. J. Rabaey, J. Ammer, J. L. da Silva Jr, D. Patel, "PicoRadio: Ad hoc Wireless Networking of Ubiquitous Low-Energy Sensor/Monitor Nodes", Proceeding WVLSI '00 Proceedings of the IEEE Computer Society Annual Workshop on VLSI (WVLSI'00), 2001. 